\title{
Atención de emergencias obstétricas en Chiapas, México. Caminando HACIA LA CONFORMACIÓN DE REDES
}

\section{Emergency Obstetric Care in Chiapas, Mexico: Moving towards the Creation of Networks}

\author{
María Guadalupe Ramírez-Rojas* \\ María Graciela Freyermuth-Enciso** \\ María Beatriz Duarte-Gómez ${ }^{* *}$ \\ DOI: http://dx.doi.org/10.29043/liminar.v19il.793
}

\begin{abstract}
Resumen: Como respuesta a las limitaciones del Sistema de Salud en México por su fragmentación y segmentación, se han puesto en marcha políticas públicas de acceso universal que operan a través de convenios y acuerdos interinstitucionales, como las dirigidas a la atención de emergencias obstétricas. A través de un estudio de caso, se ofrece un panorama de las redes de servicios de salud en Chiapas dando cuenta de su infraestructura para determinar la resolutividad real de los establecimientos ante emergencias obstétricas y su condición para operar como red colaborativa. Se muestra que las instituciones públicas del sector salud dan un uso limitado o nulo a los acuerdos y convenios interinstitucionales. Se identifican las fortalezas y obstáculos en su utilización y se efectúan recomendaciones para optimizar los mecanismos de la atención obstétrica para las mujeres en Chiapas.
\end{abstract}

Palabras clave: servicios de salud, urgencias médicas, obstetricia, políticas públicas de salud, salud maternoinfantil, hospitales.

Abstract: Responses to the systemic limitations of health care in Mexico have included public policies for universal access implemented through inter-institutional agreements. Those focused on emergency obstetric care are a vivid example of these efforts. Based on a case study, this article describes a general picture of the ways in which healthservice networks operate in the state of Chiapas. We review their infrastructure and analyze their capacity to respond to obstetric emergencies. We also assess the current condition of these networks in terms of being able to function as a collaborative network. Our findings demonstrate that public institutions in the Mexican health sector have made little or no use of the benefits offered by inter-institutional agreements, and identify the strengths and obstacles in their utilization. The results of our research can help optimize obstetric care for women in Chiapas.

Keywords: health care, health emergencies, obstetrics, public health policy, maternal and child health, hospitals.

\footnotetext{
* María Guadalupe Ramírez Rojas. Doctora en Ciencias en Salud Pública por el Instituto Nacional de Salud Pública, México. Cátedras-Conacyt, adscrita al Centro de Investigaciones y Estudios Superiores en Antropología Social (CIESAS), Unidad Sureste, México. Temas de especialización: sistemas de salud, emergencias obstétricas, salud de los pueblos indígenas, demografía. Correo electrónico: amairanai@gmail.com. ORCID: https://orcid.org/0000-0002-4251-8014

** María Graciela Freyermuth Enciso. Doctora en Antropología Social por la Universidad Nacional Autónoma de México. Profesora-investigadora en el Centro de Investigaciones y Estudios Superiores en Antropología Social (CIESAS), Unidad Sureste, México. Temas de especialización:
}

salud materna, salud de pueblos indígenas, demografía, antropología e in terculturalidad. Correo electrónico: gracielafreyermuth54@hotmail.com. ORCID: https://orcid.org/0000-0002-3871-715X

*** María Beatriz Duarte Gómez. Doctora en Ciencias de la Salud Pública por el Instituto Nacional de Salud Pública, México. Profesora-investigadora en la Universidad de Antioquía, Colombia. Temas de especialización: sistemas de salud, pueblos indígenas, envejecimiento. Correo electrónico: mbduarte52@yahoo.com. ORCID: https://orcid.org/0000-0002-3730-0811

Enviado a dictamen: 10 de febrero de 2020.

Aprobación: 25 de agosto de 2020. 
MARÍA GuAdalupe Ramírez-Rojas, MARÍA GRACIELA FREYERMUTHENCISO, MARÍA BEATRIZ DUARTE-GÓMEZ

\section{Introducción}

L as emergencias obstétricas (EO) pueden llevar a la muerte a las mujeres en el término de dos horas en el caso de una hemorragia posparto, y de dos días cuando se trata de una preeclampsia (UNFPA, 2002). Esto implica un reto para el funcionamiento de los servicios de salud, que deben asegurar un continuo en los servicios de manera oportuna y expedita desde el primer al tercer nivel de atención. Se requiere que la atención de las emergencias obstétricas (AEO) se provea con apoyo de una red de servicios conformada por un conjunto de establecimientos cuya organización y comando estén bajo una misma dirección, y que compartan recursos y logística para favorecer la circulación, en este caso, de las usuarias distribuidas en una región dada. Desde 2007, la Organización Mundial de la Salud (OMS) y la Organización Panamericana de la Salud (OPS) reconocen el papel de las redes de servicios como una estrategia que vincula las estructuras y los procesos que intervienen en la provisión de servicios, y que responde a las necesidades de la población para asegurar un acceso más justo y equitativo. Esto obedece a que la mayoría de los sistemas de salud de la región en América Latina se caracterizan por su segmentación y fragmentación, y México no es la excepción (Londoño y Frenk, 1997; Mesa-Lago, 2005). La fragmentación se refiere a que la operación de los distintos establecimientos o unidades de servicios no está incorporada en un modelo de red sanitaria que asegure la prestación de servicios de manera homogénea y continua, en términos tanto técnicos como de calidad (OPS, 2010).

La segmentación del sistema en México se reconoce en la diversidad de las instituciones públicas que integran el sector salud: la Secretaría de Salud (SSA), los Servicios Estatales de Salud (SESA), el Instituto Mexicano del Seguro Social (IMSS), el Instituto de Seguridad y Servicios Sociales de los Trabajadores del Estado (ISSSTE), Instituto el Mexicano del Seguro Social-Prospera (IMSS-P), ${ }_{1}^{1}$ Petróleos Mexicanos (PEMEX), la Secretaría de la Defensa Nacional (SEDENA) y la Secretaría de Marina-Armada de México
ATENCIÓN DE EMERGENCIAS OBSTÉTRICAS EN CHIAPAS, MÉXICO...

(SEMAR). Cada una de estas instituciones dispone de su propia red con infraestructura, financiamiento y provisión de servicios, función esta última que articula los distintos niveles de atención de la salud por medio de referencias y contrarreferencias conforme a las necesidades de las usuarias.

Las instituciones públicas de salud han firmado convenios, acuerdos y contratos de colaboración para intercambiar servicios, tales como las intervenciones diagnósticas y terapéuticas delimitadas, a fin de lograr la integración funcional del sistema. El alcance de los acuerdos y los convenios es más limitado que el de las disposiciones dictadas por Decreto Presidencial. ${ }^{2}$ Un caso paradigmático en México fue la creación del Consejo Nacional de Vacunación (CONAVA) en 1991, integrado por el Secretario de Salud y los titulares de las instituciones públicas del sector salud, lo que permitió crear el Programa de Vacunación Universal con el propósito de brindar acceso universal a todos los menores de cinco años, independientemente de su condición de afiliación al esquema básico de vacunación (Hurtado y Matías, 2005).

En 2009 se promulgó por decreto presidencial el Convenio General de Colaboración Interinstitucional para la Atención de las Emergencias Obstétricas (en adelante Convenio) (SSA, 2009), que busca el acceso universal por medio de un paquete de servicios, con el aval de los titulares de la SSA, los SESA, el IMSS, el ISSSTE y el IMSS-P. El Convenio tiene como propósito promover la prestación de servicios gratuitos y oportunos para la AEO, sin importar el tipo de afiliación institucional de las mujeres que la necesiten, y sienta las bases para otros acuerdos y convenios similares para la provisión de servicios con carácter universal en el interior del sistema de salud. El Convenio señala como requisito para la $\mathrm{AEO}$ que la brinden hospitales acreditados para ello 3 por la Dirección General de Calidad y Educación en Salud (DGCES) (SSA, 2009).

En 2011, los titulares de la SSA, el IMSS, el ISSSTE, los SESA, la Comisión Nacional de Protección Social en Salud (CNPSS), la Comisión Coordinadora de Institutos Nacionales de Salud y Hospitales de Alta Especialidad(CCINSHRAE) y la Secretaría de Salud del 
Distrito Federal (SSDF) firmaron el Acuerdo General de Colaboración para el Intercambio de Servicios (en adelante Acuerdo) (SSA, 2017). Todos los hospitales incluidos en este Acuerdo para prestar servicios de salud son del tercer nivel de atención: entre los de los SESA, se incluyen los hospitales de especialidades; entre los de la SSA, se incluyen los Institutos Nacionales de Salud, los Hospitales Regionales de Alta Especialidad (HRAE) y los Hospitales Federales de Referencia. En cuanto a los establecimientos del IMSS y el ISSSTE, participan los Hospitales Regionales, las Unidades Médicas Especializadas y los Centros Médicos Nacionales.

El Acuerdo incluye un catálogo de 715 intervenciones diagnósticas y de tratamiento para distintas especialidades médicas, de las cuales 27 están vinculadas al Convenio -22 intervenciones maternas y cinco para la atención neonatal-.

En el caso de Chiapas, existen dos hospitales regionales de alta especialidad: HRAE Ciudad Salud en Tapachula y el Hospital de Especialidades Pediátricas (HEP) en Tuxtla Gutiérrez.

Tanto el Convenio como el Acuerdo pretenden remontar las desigualdades condicionadas por la pobreza y hacer posible el acceso de las mujeres pobres a un continuo de atención, desde el primer nivel hasta los hospitales de alta especialidady, por lo tanto, prevenir la mortalidad materna en caso de necesitar AEO. Estos instrumentos de política pública se enfocan en eliminar del sistema los problemas de fragmentación y segmentación mediante la colaboración interinstitucional. Se supondría que, con la creación de estos instrumentos, las instituciones en cuestión tendrían que funcionar de manera integral como una red. El Observatorio de Mortalidad Materna (OMM) documentó que un eje clave para optimizar el funcionamiento del Convenio es la configuración de redes interinstitucionales (OMM, 2016).

El hecho de que el Convenio limite su provisión de servicios al segundo nivel de atención refleja la fragmentación del sistema de salud (OCDE, 2016). Si bien una EO amerita forzosamente la estabilización de la paciente y su referencia desde el primer nivel, el acceso al tercer nivel debería considerarse como un elemento fundamental para la atención de las complicaciones graves. En 2010, los proveedores de salud del primer nivel no conocían el Convenio ni las posibilidades de referencia interinstitucional, y cuando referían algún caso de EO, lo hacían, por lo regular, de manera intrainstitucional (CIESAS e INMUJERES, 2010).

Se ha documentado que una parte de las mujeres urbanas dispone de una infraestructura y una información privilegiadas en comparación con las mujeres rurales, pues conocen su derecho a recibir AEO en cualquier establecimiento de salud, sin importar su afiliación (Ramírez y Freyermuth, 2013), así como la ventaja -y la oportunidad - de acudir directamente al segundo o al tercer nivel de atención. Los retos son disminuir las brechas sociales entre el ámbito urbano y el rural, reparar las asimetrías de información entre las mujeres mexicanas y asegurar la atención oportuna de las complicaciones mediante una red de servicios eficiente. El Convenio no hace explícita la figura de las redes de servicios; no obstante, su configuración aseguraría la colaboración interinstitucional como herramienta para superar la fragmentación y segmentación del sistema a fin de asegurar el acceso universal de las mujeres a la AEO. Interesa, por tanto, saber si el principio de acceso universal implícito en el Convenio y el Acuerdo logra instrumentarse, y si con ello se reducirían o se eliminarían las desigualdades ocasionadas por la segmentación y la fragmentación de los servicios.

En este artículo se analizan la organización de los servicios de salud disponibles en Chiapas y su coordinación como red para la atención de las EO, a la luz de convenios y acuerdos entre instituciones de salud, y que posibilitarían un continuo de la atención. Estudiar la red de servicios de atención a las EO en Chiapas como estudio de caso puede dar cuenta de la situación actual y de las necesidades y los retos que habrán de afrontar las redes que aspiren a reorganizarse funcionalmente.

\section{Material y métodos}

Se trata de un estudio exploratorio descriptivo, ambispectivo, cuali-cuantitativo, y se apoyó para su diseño en el estudio de caso para el análisis de la red de servicios de atención de las EO en Chiapas, a fin de estudiar la 
María Guadalupe Ramírez-Rojas, María GRACIELA FREYeRMUTHENCISO, MARÍA BEATRIZ DUARTE-GÓMEZ

provisión de AEO a partir de la colaboración interinstitucional a través del Convenio y el Acuerdo.

Se optó por hacer un estudio de caso de los denominados críticos, que se distinguen por su decisiva correspondencia con el problema, a fin de corroborar o descartar las hipótesis de las cuales se parte (Flyvbjerg, 2003). Interesaba reconocer si el Acuerdo y el Convenio facilitan la coordinación y operación de las redes de servicios de Chiapas para otorgar un continuo de la AEO materna y neonatal. El estudio de caso permite recabar sistemáticamente información de la realidad social de los procesos significativos de las redes de servicios que brindan AEO. Este abordaje permite comprender mejor el fenómeno de nuestro estudio, e identificar los factores que limitan y los que facilitan la AEO oportuna de mujeres y neonatos. La unidad de análisis la constituyen las redes de servicios de salud en Chiapas, que posibilitan el acceso a la AEO a través del Convenio y el Acuerdo.

La selección de Chiapas como espacio del presente caso obedeció a la importancia, en razón de su relevancia social, ya que en este estado ocurre una de las razones de mortalidad materna (RMM) más elevadas de México, de 65 muertes en 2016, lo que representó una RMM de 58.3 por cada 100000 nacidos vivos (OMM, 2018). Por este hecho, puede considerarse paradigmático. La temporalidad corresponde a los años de 2010 a 2015.

Como señala Giménez, citando a Yin, un estudio de caso es un fenómeno de la vida real que se investiga empíricamente de manera contemporánea en su contexto, con el fin de reconocer sus límites (Yin, 2009 en Giménez, 2012), ya que se persigue la interpretación del fenómeno, entendido este como la posibilidad o no de la provisión de la AEO a través de la colaboración interinstitucional entre las distintas redes de servicios que integran el estado de Chiapas.

Interesa reconocer el grado de involucramiento entre las distintas instituciones a partir del Convenio y el Acuerdo, así como los posibles reductos que limiten su utilización, identificar los contextos y problemáticas, o corroborar si estos instrumentos cumplieron con su función explícita de eliminar la segmentación y la fragmentación propias del sistema de salud.
Nos apoyamos en información documental proveniente de diversas fuentes, tanto primarias como secundarias. Respecto a fuentes de tipo primario, fueron el producto del trabajo de campo desarrollado en monitoreos realizados con apoyo del OMM de México. Dichos monitoreos estuvieron enfocados al análisis del Convenio y se desarrollaron en el ámbito hospitalario en 2011, 2013 y 2015. Se dispuso de información proveniente de entrevistas abiertas y semiestructuradas al personal de salud y a los responsables de la toma de decisiones a nivel institucional, listas de cotejo y guías de recorrido, aplicadas en establecimientos del ámbito hospitalario. Se utilizaron las bases de datos de la SSA correspondientes a la Dirección General de Información en Salud (DGIS) e información solicitada a la Dirección General de Planeación y Desarrollo en Salud (DGPLADES), dependencia encargada de la operación del Convenio.

Para el estudio de la red de servicios se consideraron las recomendaciones de organizaciones internacionales para los establecimientos que presten servicios de AEO básica e integral (OMS et al., 2011). Una red de servicios posibilita otorgar atención desde el primer nivel hasta el tercer nivel de atención, lo que implica capacidades resolutivas diferenciales de atención de las EO. Para clasificar los establecimientos de acuerdo con su capacidad resolutiva, se consideró la definición de la AEO planteada por la SSA. Como propuesta de las autoras, se añadió la capacidad resolutiva alta complementaria, en la que se incluyen los establecimientos de alta especialidad que no están considerados en el Convenio. Este nivel debería asegurar el continuo de la atención entre el segundo y el tercer nivel a las mujeres con complicaciones obstétricas y a los neonatos, conforme a lo señalado en el Acuerdo y el Convenio. Así, se consideró la capacidad resolutiva básica, media, alta y alta complementaria para la AEO. ${ }^{4}$ A aquellos establecimientos no incluidos en el Convenio se les denominó no acreditados.

A partir de estas dos propuestas (organismos internacionales y SSA), y para los fines analíticos de este estudio, se consideraron como elementos necesarios para cada establecimiento, tanto en el plano local como en el sistémico, los siguientes componentes para 
la operación en red: a) disponibilidad de sistemas de referencia y contrarreferencia, b) capacidad resolutiva en red: disponibilidad para resolver las EO en colaboración, y c) disponibilidad de terapia intensiva: para adultas y neonatal.

Para analizar la red, esta investigación se sirvió de la definición conceptual de Gillies y colaboradores, quienes señalan que las redes de servicios de salud son aquellas que convergen entre sí, cumplen con estándares de operación, información de sistemas y otros recursos logísticos para coordinarse funcionalmente entre los diversos niveles e instituciones dentro de una determinada zona geográfica, y las comanda un operador común (Gillies et al., 1993).

Una red para la AEO se concebirá como una organización creada por los diversos establecimientos de las instituciones que la integran y que tiene por objetivo común dar acceso universal a la AEO. Su mando rector único recae en la SSA. El Convenio y el Acuerdo establecen los mecanismos de coordinación que regulan la colaboración intrainstitucional, y el Sistema de Registro de la Emergencia Obstétrica (SREO) constituye su sistema de rendición de cuentas. El SREO es la plataforma donde los hospitales acreditados para brindar AEO reportan los casos atendidos. Una red para la AEO operaría en un territorio definido, con amplio conocimiento de las necesidades de cada uno de los miembros que integran la red (ver Figura l).

La investigación se sirvió de un sistema de posicionamiento geográfico (mapas de Google) para analizar la distancia y el tiempo de recorrido por carretera entre los distintos establecimientos que participaron en la referencia de pacientes con EO, y de información recopilada a través de entrevistas a directivos de los distintos establecimientos hospitalarios, quienes corroboraron los tiempos y distancias de recorrido. ${ }^{5}$ Se identificaron hospitales que concentraron referencias de cuatro o más hospitales, y a los que se denominó establecimientos concentradores.

Para describir la información, se consideró si las referencias de los establecimientos concentradores de la AEO se apegaban a lo señalado en el Convenio: si el establecimiento de origen: a) se ubicaba a la distancia y el tiempo recomendados, y b) tenía una capacidad resolutiva menor que la del establecimiento receptor. En aquellos casos en que las referencias se hicieran al margen de las recomendaciones del Convenio, se empleó la expresión "salto de establecimiento(s)" para aludir a las referencias en que, en la búsqueda de la atención, no se consideró un hospital con capacidad resolutiva para la $\mathrm{AEO}$ en la ciudad más próxima, y se pasó de largo para acudir directamente al hospital receptor elegido.

Para el análisis y descripción de las redes nos apoyamos del software UCINET 6.707 y NetDraw, con la finalidad de facilitar su representación por medio de grafos que diesen cuenta de los vínculos, y la dirección de la punta de la flecha indica la concurrencia de la recepción o envío de referencias por AEO.

\section{Estudio de caso: redes de servicios de Chiapas}

\section{a) Disponibilidad de sistemas de referencia y contrarreferencia}

En México, los ejes vinculantes de la política de acceso universal a la AEO son el Convenio y el Acuerdo, los cuales toman como punto de partida el segundo y el tercer nivel de atención, excluyendo el primer nivel, que es la puerta de entrada principal al sistema de salud. Cabe recordar aquí que casi el 75 \% de la atención de las afecciones de la población se brinda a mujeres embarazadas y a niños, y que el $95 \%$ de las unidades de salud del país — alrededor de 13000 — son de atención primaria (DGED, 2009).

El primer nivel de atención está constituido por centros de salud que se encargan del control y la vigilancia de los embarazos de bajo riesgo y de la detección temprana de riesgos obstétricos. Si el embarazo de una mujer se considera de alto riesgo, debe referirse al segundo nivel de atención para su valoración. El primer nivel de atención no tiene la capacidad resolutiva necesaria para dar AEO, pues carece de personal capacitado y tiene limitaciones en infraestructura, insumos, equipo médico y ambulancias, aunque se ha señalado que puede ser fundamental para la estabilización de esta (OMS et al., 2011). Sin embargo, en México este nivel no se considera para brindar AEO inmediata y vigilar su 
estabilización. La estrategia de AEO en el primer nivel se limita a canalizar a la paciente a una unidad hospitalaria, y es responsabilidad de ella y su familia acudir al hospital. Esto refleja el limitado papel del primer nivel de atención en la red de servicios ante una EO.

En el segundo nivel de atención predomina la atención ginecoobstétrica especializada. Los hospitales generales (HG) concentran las cuatro especialidades básicas: ginecoobstetricia, pediatría, medicina interna y cirugía general, y disponen de infraestructura y equipamiento de quirófanos, banco de sangre o puesto de sangrado, y servicio de laboratorio e imagenología, entre otros, con lo que completan la prestación de servicios de salud.

En el tercer nivel se brinda atención a padecimientos que ameritan procedimientos especializados y equipo de alta tecnología. Para lograr un continuo en la atención, se siguen los procedimientos médicos administrativos ${ }^{6}$ de referencia y contrarreferencia, que permiten la coordinación entre los distintos niveles, tanto para resolver situaciones de urgencia como para obtener evaluaciones diagnósticas de personal médico especializado (Diario Oficial de la Federación, 2012) (ver Figura 2).

La AEO recae principalmente en el segundo nivel, mediante las estrategias del Convenio y de Embarazo Saludable ${ }^{7}$ para mujeres sin seguridad social. El primer nivel de atención no participa en la AEO, y el tercer nivel lo hace de manera limitada, aunque se tiene prevista en el Acuerdo.

El Convenio señala los siguientes tres criterios para referir EO de un establecimiento a otro: 1) la unidad médica que solicita la referencia no dispone de la capacidad resolutiva suficiente para brindar AEO, y el establecimiento al que se hace la referencia dispone de mayor capacidad resolutiva; 2) el establecimiento al que se hace la referencia es más accesible y cercano para la usuaria, considerando una distancia de 70 kilómetros como máximo o una hora y media de traslado entre ambos hospitales, y 3) la referencia se hace una vez estabilizada la EO, y para el traslado se dispone del apoyo de una ambulancia en que la mujer viaja en compañía de personal médico y un familiar.
El Convenio también especifica las condiciones en las que no se hacen traslados: 1) en cualquier situación que ponga en riesgo la vida de la mujer, como ocurre con las pacientes inestables; 2) cuando el riesgo de traslado a otro hospital es mayor que el beneficio; 3 ) cuando el traslado dure más de dos horas, y 4) cuando el hospital que pretende hacer el traslado dispone de una capacidad resolutiva igual o mayor, o dispone de terapia intensiva, a diferencia del hospital al que pretende referir a la usuaria.

Se identificaron variantes en los criterios que rigen los procesos de referencia por institución para responder a una EO en Chiapas que se describen a continuación

Red de servicios del IMSS. La integran cuatro establecimientos acreditados como de capacidad resolutiva media. Dos están incluidos en el Convenio. Los establecimientos incluidos en el Convenio no referían las EO a ningún otro centro, excepto cuando las pacientes ameritaban el servicio de alguna especialidad del tercer nivel de atención, y solo si se trataba de una paciente estable que fuera derechohabiente del IMSS. Los traslados se hacen por vía aérea a la Ciudad de México, en donde se envían ya sea al Centro Médico Nacional (CMN) Siglo XXI o al CMN La Raza; en promedio, se realizan de tres a cinco traslados al año por causas maternas -monitoreos OMM de los años 2011, 2013, 2015-. Puesto que los traslados se hacen en líneas aéreas comerciales, en sus horarios establecidos - suele haber uno o dos vuelos diarios-, algunas referencias pueden demorarse. Los dos establecimientos acreditados reciben referencias de hospitales rurales del IMSS-P, de mujeres que pertenecen al universo de trabajo ${ }^{8}$ de dicha institución. En 2006, el IMSS y el IMSS-P firmaron el Acuerdo 126, mediante el cual los hospitales del segundo y el tercer nivel de atención del IMSS atenderían EO en beneficiarias del IMSS-P. La atención se brindaría de manera inmediata y gratuita, y la cubriría el IMSS-P (IMSS, 2006). Entre 2013 y 2015 no recibieron pacientes del Instituto de Salud del Estado de Chiapas (ISECH) o del ISSSTE.

Red de servicios del ISSSTE. Está conformada por un hospital acreditado con capacidad resolutiva alta y tres 
establecimientos hospitalarios de capacidad resolutiva media no acreditados. No hay prácticamente referencias interinstitucionales. Por excepción, alguna mujer no afiliada acude espontáneamente a solicitar AEO —en promedio, una o dos al año- La red dispone de unidades de medicina familiar (UMF) o consultorios del primer nivel de atención con servicio de urgencias que operan, en su mayoría, en los turnos matutino o diurno, de lunes a viernes. Puesto que las UMF no disponen de ambulancia, las mujeres con EO acuden directamente al hospital más cercano del IMSS-P. De ahí, una vez estabilizadas, son trasladadas en ambulancia del IMSS-P a los hospitales del ISSSTE. Cuando el caso lo amerita, se refiere a la mujer o al neonato a un hospital de alta especialidad de la SSA —al HEP de Tuxtla Gutiérrez y al HRAE de Ciudad Salud, Tapachula-. Estas referencias se hacen en virtud de un convenio directo entre el ISSSTE y la SSA, al margen del Convenio y el Acuerdo. Un acuerdo interno con el HG Pascasio Gamboa (Tuxtla Gutiérrez), del ISECH, otorga pase directo a la Unidad de Cuidados Intensivos Adultos (UCIA) del hospital a las mujeres trabajadoras del ISSSTE, o a sus familiares, de presentar una EO.

Red de servicios del ISECH. Está conformada por once hospitales acreditados para brindar AEO: cuatro de capacidad resolutiva alta y siete de capacidad resolutiva media. El ISECH dispone de catorce establecimientos no acreditados, dos de ellos de capacidad resolutiva alta complementaria, uno de capacidad resolutiva alta, uno de capacidad resolutiva media y cinco de capacidad resolutiva básica. Las referencias se concentran, en su mayoría, en los establecimientos de más alta capacidad resolutiva y que disponen de terapia intensiva o de banco de sangre. El mayor número de referencias interinstitucionales a los establecimientos del ISECH proviene del IMSS-P, puesto que desde 2011 parte de su población es beneficiaria del Seguro Popular (SP) (IMSS, 2016).

Algunas veces, un establecimiento de mayor capacidad resolutiva hace la referencia hacia uno de capacidad resolutiva menor. Por ejemplo, el HR Dr. Rafael Pascacio Gamboa (Tuxtla Gutiérrez), de capacidad resolutiva alta, refiere mujeres a establecimientos de capacidad resolutiva media para la atención de abortos sin com- plicaciones. Se documentó que cuando no es posible hacer la referencia en la propia entidad (tres o cuatro veces al año), se hace a hospitales de alta especialidad de los SESA de Tabasco.

Las únicas referencias interinstitucionales del ISECH fueron a hospitales de alta especialidad de la SSA, en virtud de un convenio estatal — no por la vía del Convenio o del Acuerdo- - para evitar que los pacientes incurriesen en gastos catastróficos como resultado de que el SP no cubría algunos servicios de su cartera, como los estudios diagnósticos y los quirúrgicos.

Red de servicios del IMSS-P. Está constituida por diez establecimientos hospitalarios incluidos en el Convenio. Nueve son de capacidad resolutiva media y uno es de capacidad resolutiva baja. Se documentaron dos tipos de referencias según la gravedad y el estado de las pacientes:

1. Para beneficiarias del IMSS, la referencia se apoyaba principalmente en el Acuerdo 126, ante una EO que ameritara cuidados especializados. De necesitar terapia intensiva - ya fuera la madre o el neonato-, se hacía la referencia a algún establecimiento del ISECH; de no concretarse por motivos de saturación hospitalaria, y solo así, se gestionaba al IMSS. Se documentó que la referencia de la mujer podía demorar de uno a dos turnos — de 8 a 12 horas - y del neonato, de 2 a 15 días, o nunca realizarse.

2. Las mujeres no pertenecientes al universo de trabajo del IMSS-P se referían a la institución a la que estuvieran afiliadas. Las afiliadas al SP se referían a hospitales del ISECH, y las afiliadas a la seguridad social, a hospitales del IMSS o del ISSSTE. Los neonatos que ameritaban tratamiento quirúrgico o de subespecialidad se referían al HEP de Tuxtla Gutiérrez de la SSA, previo análisis del caso.

Red de Hospitales Regionales de Alta Especialidad de la SSA. Está constituida por el HRAE Ciudad Salud en Tapachula y el HEP de Tuxtla Gutiérrez. Los HRAE de la SSA no hacen ningún tipo de referencia puesto que son los de mayor capacidad resolutiva en la entidad. Ofrecen servicios de subespecialidades y de terapia 
intensiva. Cuentan con un Servicio de Referencia y Contrarreferencia para recibir pacientes de otras instituciones tras evaluar si las mujeres o los neonatos son candidatos de atención. Los hospitales de origen deben enviar un resumen clínico vía fax, adjuntar los estudios de laboratorio y gabinete y comunicarse por teléfono para confirmar su recepción. Los HRAE tardan entre ocho horas y dos días en responder, y reciben urgencias solo en casos excepcionales. Los HRAE de la SSA tienen un convenio particular con el ISSSTE de Chiapas para la atención de pacientes que necesiten sus servicios. Basta con que informen de la referencia del paciente, pues los casos no se evalúan, por lo que puede mantenerse un continuo en la AEO. Hubo, en promedio, de dos a tres referencias interinstitucionales al año documentadas en el SREO.

Los estudios realizados en Chiapas con apoyo del OMM en 2011, 2013 y 2015 sobre las redes de servicios documentan la ausencia de redes interinstitucionales de servicios para la AEO, ya que las instituciones que integran el sector —el ISECH, el IMSS, el ISSSTE y el IMSS-P - privilegian la referencia de las EO al interior de sus propias redes de servicios, sin considerar la distancia o el tiempo de traslado, e incluso se apoyan en otras unidades hospitalarias de su misma red ubicadas en entidades federativas circunvecinas. Pero no se considera la colaboración entre diferentes instituciones ubicadas en la misma ciudad o entidad.

\section{b) Disponibilidad de terapia intensiva para adultas y neonatal}

La sobrevivencia de las mujeres y neonatos ante una $\mathrm{EO}$ frecuentemente depende de la posibilidad de ingresar a terapia intensiva. En la UCIA y en la Unidad de Cuidados Intensivos Neonatales (UCIN) ingresan solo pacientes en estado crítico que padecen una disfunción orgánica única (la disfunción de un órgano vital) o múltiple (la disfunción de dos o más órganos vitales). Un criterio para catalogar un establecimiento como de capacidad resolutiva alta para la $\mathrm{AEO}$ es que disponga de una UCIA y una UCIN.

De los 24 hospitales de Chiapas que participan en el Convenio, cinco están acreditados con capacidad resolutiva alta. Si se triangulan varias fuentes (SSA, OMM), se encuentra que, en Chiapas, la disponibilidad de camas en UCIA es de 49; en UCIN de 35, y en terapia intermedia, de 19 (de las cuales seis son para neonatos). Hay, además, unas diez camas en un área acondicionada donde se brinda atención equivalente a la de una UCIN, pero sin los requisitos y especificaciones normadas. Algunos hospitales que no disponen de servicios de cuidados intensivos cuentan con equipamiento y recursos humanos especializados, ya sea de base o subrogados. Incluyendo estas modalidades de atención, se obtiene un total de 23 camas acondicionadas para brindar temporalmente AEO y monitorear a las mujeres que ameriten vigilancia continua.

Si bien el Convenio señala que los hospitales del ISECH, de capacidad resolutiva alta, cuentan con UCIA y UCIN, en la práctica disponen solo de una de estas o de ninguna.

En cuanto a los establecimientos del ISECH: el HG Villaflores no dispone de ningún tipo de terapia por falta de recursos humanos a pesar de tener asignadas, desde su inauguración en 2015, las áreas correspondientes. Los Hospitales de la Mujer en San Cristóbal de Las Casas y en Comitán disponen solo de UCIN. En Tuxtla Gutiérrez, el Hospital de Especialidades (HE) Gilberto Gómez Maza, inaugurado también en 2015, recibió la UCIA que se desmanteló del Hospital Regional (HR) Dr. Rafael Pascacio Gamboa, junto con el personal médico especializado en medicina del enfermo en estado crítico, medicina interna y del resto de subespecialidades.

Respecto a los establecimientos de seguridad social: el HG Belisario Domínguez, del ISSSTE (Tuxtla Gutiérrez), dispone únicamente de UCIA, y aunque tiene asignada un área para UCIN, no cuenta con personal calificado. Los dos establecimientos del IMSS —uno en Tuxtla Gutiérrez y el otro en Tapachula- están clasificados como de capacidad resolutiva media para la AEO, pero deberían estarlo como de capacidad resolutiva alta puesto que disponen de una UCIA y una UCIN; en la cédula de acreditación del Convenio informan solo de la UCIA, porque llaman "cunero patológico" al área que corresponde a la UCIN, si bien prestan en ella servicios de terapia neonatal (ver Tabla l). 
Se identificó, así, que no hay UCIA o terapia intermedia exclusiva de obstetricia. Solo los dos hospitales del IMSS disponen de al menos una cama para obstetricia. La terapia intermedia se reserva para la atención de pacientes con enfermedades agudas no críticas que ameritan seguimiento y cuidados especializados, pero cuyo estado no es crítico o grave (Dávila, 1970). Disponer de terapia intermedia acortaría el tiempo de estancia hospitalaria de las pacientes que no requieren terapia intensiva, aunque - de acuerdo con lo documentado- mujeres candidatas a ser atendidas en la UCIA se atienden en cuidados intermedios por la limitada disponibilidad de espacio en la UCIA. Se identificó también que en los hospitales que no disponen de UCIA ni de terapia intermedia se usa el área de choque en urgencias o el Servicio de Recuperación de las áreas quirúrgicas, para vigilar a las pacientes en estado crítico cuando no es posible referirlas a otra unidad hospitalaria que disponga de servicios especializados.

Proveedores de salud del IMSS mencionaron que cuando las mujeres no tienen seguro médico y son atendidas por una EO, una vez estabilizadas son referidas de inmediato a su institución de afiliación o a hospitales del ISECH, con lo que podrían ser referidas a establecimientos que no dispongan de UCIA o de terapia intermedia, aun si el padecimiento ameritara vigilancia a más largo plazo. El IMSS priorizaba, por lo general, la atención de sus derechohabientes. De tratarse de mujeres no afiliadas, no se esperaba a que se cumpliese el plazo de estancia máxima de diez días que cubre el Convenio ni se utilizaba el Acuerdo, ya que lo desconocían, lo que refleja algunas de las barreras en coordinación para asegurar el continuo de la AEO.

La situación se complica para los neonatos porque el tiempo de estancia en una UCIN puede ser mayor. Por ejemplo, un recién nacido con diagnóstico de prematuridad extrema debe permanecer en la UCIN un promedio de 49 días (Tello, 2007). Se documentó que la referencia de un neonato prematuro de un hospital a otro podría demorar de dos a quince días o no hacerse en absoluto dado el limitado espacio de las UCIN disponibles. Un recién nacido prematuro necesita apoyo ventilatorio, monitor y al menos una bomba de infusión para la aplicación de medicamentos. Los establecimientos del IMSS-P (ubicados en el medio rural) disponen de solo uno o dos ventiladores que podrían emplearse en neonatos, en pediatría o en adultos, por lo que pueden no estar disponibles.

En los hospitales básicos comunitarios que no cuentan con ventiladores ni con bombas de infusión se realizan maniobras de ventilación manual, siempre y cuando se disponga de médicos internos de pregrado, por lo que los neonatos pueden fallecer antes de recibir cuidados especializados.

De acuerdo con la cédula de acreditación para la AEO, a fin de garantizar la seguridad en la atención de las pacientes los establecimientos con capacidad resolutiva alta deben disponer de personal calificado en medicina del enfermo en estado crítico las 24 horas de los 365 días del año (DGCES, 2013).

La Norma Oficial Mexicana para la organización y funcionamiento de las unidades de cuidados intensivos (Diario Oficial de la Federación, 2013) establece que se disponga de al menos un intensivista certificado a cargo de la terapia o un especialista en medicina interna, neumología, cardiología u otra rama, que demuestre documentalmente haber cursado estudios en medicina del enfermo en estado crítico avalados por una institución de salud reconocida. El personal de enfermería, por su parte, debe acreditar la especialidad o tener un diploma que avale su entrenamiento y capacitación en el manejo de pacientes de la UCIA o la UCIN, según sea el caso.

La disponibilidad de intensivistas era del cien por ciento, en el HEP de Tuxtla Gutiérrez, y en el HRAE de Ciudad Salud, en Tapachula, ambos de la SSA, con capacidad resolutiva alta complementaria, establecimientos no incluidos en el Convenio y considerados como no acreditados. En contraparte, se documentó que hospitales acreditados en el Convenio como el HR Dr. Rafael Pascacio Gamboa (Tuxtla Gutiérrez), del ISECH, disponían solo de un intensivista por las mañanas entre semana en terapia intermedia, y de neonatólogos en todos los turnos en la UCIN. El HG Tapachula, del ISECH, con capacidad resolutiva alta, no dispone de intensivistas; cuenta solo con un neo- 
MARÍA GuAdalupe Ramírez-Rojas, MARÍA GRACIELA FREYERMUTHENCISO, MARÍA BEATRIZ DUARTE-GÓMEZ

natólogo por las noches y durante las jornadas diurnas especiales de fin de semana.

La UCIA del HG Belisario Domínguez, del ISSSTE, es atendida por un especialista por las mañanas de lunes a viernes, y la UCIN no dispone de neonatólogo, por lo que casi siempre refieren a los neonatos al IMSS o al HEP Tuxtla Gutiérrez mediante convenios distintos del Convenio. En todos los casos mencionados donde no se disponía de personal especializado en los cuidados del enfermo en estado crítico, eran médicos adscritos al servicio de medicina interna o pediatría quienes, además de cumplir con sus labores, supervisaban las terapias y daban atención a solicitud del personal de enfermería.

El HEP y el HRAE de la SSA, ambos con capacidad resolutiva alta complementaria, disponían de enfermeras especialistas en el cuidado de pacientes adultos en estado crítico o en neonatología. Los hospitales del IMSS y el ISSSTE contaban con enfermeras especialistas al menos en los turnos matutinos, vespertinos y entre semana. Los hospitales del ISECH disponían de enfermeras especialistas en los turnos diurnos entre semana; estas enfermeras rotan cada seis meses por los distintos servicios. Las enfermeras especialistas suelen ocupar cargos administrativos, en ocasiones directivos. Este punto muestra las barreras en la organización y las limitaciones en la red respecto de la oferta adecuada de servicios; tales limitaciones impiden dar continuidad a la atención y podrían resumirse en que: los hospitales acreditados no siempre cuentan con UCIA y UCIN; no se dispone para todas las terapias intensivas de personal calificado las 24 horas durante los 365 días del año, como se estipula en la normativa; los hospitales no siempre están catalogados con su verdadera capacidad resolutiva; los hospitales improvisan espacios inadecuados para la atención de pacientes que requieren cuidados intensivos; la limitada presencia de camas de cuidados críticos determina las altas prematuras de enfermas que requieren de cuidados especializados, sobre todo de aquellas que fueron atendidas en instituciones a las que no están afiliadas, y la necesidad de UCIN se evidencia ante el retardo de ubicar a los neonatos que las requieren antes de fallecer.
ATENCIÓN DE EMERGENCIAS OBSTÉTRICAS EN CHIAPAS, MÉXICO...

\section{c) Capacidad resolutiva en red: disponibilidad para colaborar en la AEO}

En la Figura 3 se detalla la ruta de las referencias de las mujeres con EO de los distintos establecimientos de salud de Chiapas, lo que da cuenta de las realizadas intra e interinstitucionalmente (ver Figura 3). Se muestra cómo intervienen barreras organizativas al interior de las instituciones y cómo la oferta de servicios para la AEO determina la búsqueda según la atención especializada disponible. Se documentaron los procesos de referencia de las EO a partir de la identificación de los hospitales considerados como concentradores - catorce-, los cuales recibían referencias de al menos cuatro o más establecimientos hospitalarios, entre los que se encuentran HRAE, hospitales del tercero y segundo nivel de atención e incluso básico comunitarios y rurales (ver Tabla 2).

Entre el $29 \%$ y el $100 \%$ de las referencias recibidas por los hospitales concentradores superaban el tiempo o la distancia recomendados en el Convenio, y se documentó que, en el proceso de referencia, las ambulancias llegaban a saltarse uno o más establecimientos resolutivos. Esto se debe a lo ya descrito en el componente de los sistemas de referencia, que las distintas instituciones guían los procesos de referencia al interior de sus propias redes de servicios y solo de manera limitada o por excepción hacen referencias interinstitucionales, sin atender las recomendaciones del límite establecido en el Convenio relativas a la distancia y el tiempo para referir las AEO y garantizar la seguridad de las mujeres.

Nueve de los establecimientos identificados como concentradores (64\%) recibían referencias de establecimientos con igual capacidad resolutiva, contraviniendo lo establecido en el Convenio. Seis de los hospitales concentradores (43\%) recibían referencias de establecimientos con una mayor capacidad resolutiva y, de estos, el $67 \%$ son hospitales con una capacidad resolutiva media, que reciben referencias de hospitales de capacidad resolutiva alta.

Los establecimientos que no recibían referencias de otros hospitales de mayor capacidad resolutiva 
fueron los HRAE, los hospitales de especialidades con capacidad resolutiva alta complementaria y establecimientos con capacidad resolutiva alta (57\%), en virtud de que disponen de la mayor capacidad resolutiva existente en la entidad, con excepción de los hospitales del ISECH acreditados para la AEO: el HR Dr. Rafael Pascacio Gamboa, en Tuxtla Gutiérrez — recibe casos de los dos HRAE de la entidad, en Tapachula y Tuxtla Gutiérrez, y del HG Belisario Domínguez del ISSSTE, en Tuxtla Gutiérrez-, y el HG de Tapachula, que reciben casos del HRAE Ciudad Salud, ubicado en la misma ciudad.

Todos los hospitales concentradores reciben referencias de establecimientos con una menor capacidad resolutiva a excepción del HR Venustiano Carranza, del IMSS-P, que recibe referencias del segundo nivel de atención correspondientes a hospitales con igual o mayor capacidad resolutiva.

Todos los establecimientos concentradores recibían referencias que, en el trayecto previo a su llegada, habrían realizado al menos un salto de algún otro establecimiento con capacidad resolutiva para la AEO. Los hospitales a los que se refirieron las EO saltando hospitales más cercanos con posibilidades de atención fueron: HEP Tuxtla Gutiérrez de la SSA (89 \%), HBC Acala del ISECH (82 \%) y HR Venustiano Carranza del IMSS-P (80\%).

Estas descripciones se complementaron, finalmente, con información de si los establecimientos recibían o no referencias interinstitucionales o interestatales. Los establecimientos del ISECH fueron los únicos en recibir referencias interestatales, provenientes de los SESA de Tabasco y Oaxaca.

El caso del HR Venustiano Carranza, del IMSS-P (identificado en la Figura 3 con el número 10), permite ofrecer un ejemplo ilustrativo de las dinámicas de operación de las redes en Chiapas. Este hospital es de capacidad resolutiva media (ver Tabla 3) y recibe referencias de dos hospitales de capacidad resolutiva alta -el HR Dr. Rafael Pascacio Gamboa, de Tuxtla Gutiérrez, y el HG María Ignacia Gandulfo de Comitán, ambos con terapia intensiva-, así como del HG Villaflores. Las referencias que recibe para la AEO son de mujeres que necesitan manejo quirúrgico, pero no cuidados especializados - para abortos y cesáreas no complicadas-, aunque las distancias rebasan lo señalado por el Convenio. Recibe EO del HBC Acala — que se considera como establecimiento estabilizador-y del HBC Ángel Albino Corzo, que es el más lejano de los hospitales que les refieren pacientes, con lo que se reflejan las barreras geográficas que impiden apegarse a lo estipulado en el Convenio en cuanto al tiempo y la distancia de traslado.

\section{Discusión}

En un estudio desarrollado en Colombia, Herrera, Conde y Pinzón (2017) describieron una red integrada por dos instituciones hospitalarias y evaluaron indicadores de referencia relativos al tiempo promedio de remisión de pacientes con convenio y sin convenio. En situaciones de urgencia y no urgentes, se evidenció una falta de oportunidad y demora en virtud de trámites administrativos derivados de respuestas de los aseguradores adscritos a convenios, lo que presentaba problemas en el sistema de referencia y contrarreferencia y en la continuidad de la atención en aquellos pacientes que no tenían convenios (Herrera, Conde y Pinzón, 2017). En el contexto colombiano, las redes integrales de prestadores de servicios de salud son definidas por la articulación de diversos prestadores de servicios del sector público y privado - denominadas Instituciones Prestadoras de Salud (IPS) y Entidades Promotoras de Salud (EPS) - , organizados funcionalmente por territorios definidos de operación. Al igual que en México, están fragmentados y segmentados, pero la participación privada es mayor por medio de convenios que les acreditan con el fin de satisfacer demandas no satisfechas por el Estado (MINSALUD, 2016).

El caso de México es paradigmático dada su estructura fragmentada y segmentada. El Convenio y el Acuerdo pretendían ser la punta de lanza de la integración funcional del sistema, ya que el Convenio abrió la puerta por vez primera a la homologación de tarifas hospitalarias entre instituciones del sector público y sentó las bases para futuros convenios de 
MARÍA GuAdalupe Ramírez-Rojas, MARÍA GRACIELA FREYERMUTHENCISO, MARÍA BEATRIZ DUARTE-GÓMEZ
ATENCIÓN DE EMERGENCIAS OBSTÉTRICAS EN CHIAPAS, MÉXICO... corte similar que podrían no solamente otorgarse bajo la tutela de instituciones públicas, sino también con la inclusión del sector privado. Un ejemplo de ello es la denominada Ley de Asociación Público Privada, que se rige bajo el principio de desarrollar proyectos que complementen los servicios públicos cuando el Estado no disponga de suficiente infraestructura y posibilidad de proveerlos, por lo que otorga una concesión de estos a las instancias privadas (Diario Oficial de la Federación, 2018), como lo muestra la operación de los HRAE y diversas Unidades Médicas Especializadas (UMAE) bajo este modelo de gestión.

A diferencia del modelo colombiano, cuyo funcionamiento es ineficiente por trámites administrativos, en el caso de Chiapas, en el funcionamiento de la red de servicios de salud las referencias de las instituciones se dirigen, en primera instancia, a sus propias redes de servicios sin importar si la distancia o el tiempo de traslado están previstos en el Convenio. Se producen incluso hacia otras entidades sin previa solicitud del apoyo interinstitucional en la propia ciudad o entidad, lo que refleja barreras organizativas que impiden a la red remontar las barreras geográficas y económicas.

El Convenio y el Acuerdo se emplean limitadamente o no se emplean en absoluto. Las referencias interinstitucionales se realizan en virtud de convenios anteriores - como el Acuerdo 126 entre el IMSS y el IMSS-P-o de convenios interinstitucionales dentro de cada estado, como es el caso del Hospital Regional de Alta Especialidad de la Mujer en Tabasco, donde se cuenta con diferentes convenios de subrogación con diversas instituciones -ISSSTE, IMSS, SEDE$\mathrm{NA}-\mathrm{y}$ con diferentes tarifas cuyo pago se realiza al final del mes. Por ello, pese a existir el Convenio se utilizaban convenios anteriores porque consideran tarifas más altas y disponibilidad de los pagos en un menor tiempo (OMM, 2011), lo cual evidencia la necesidad de un mayor fortalecimiento del papel de rectoría de la SSA.

Uno de los obstáculos en la puesta en marcha del Convenio y el Acuerdo, que supone una normativa general y que persigue su aplicación universal en las instituciones que integran el sector, es la presencia de convenios anteriores que brindan AEO y que desvirtúan esta política pública, cuyo objetivo primordial a largo plazo es la integración funcional del sistema.

El Convenio y el Acuerdo aluden implícitamente a la colaboración interinstitucional. Es necesario explicitar y formalizar cómo derivar pacientes ante una EO con criterios homologados para todas las instituciones que integran el sector salud. Una forma operativa es la creación de redes interinstitucionales por parte de los encargados federales del Convenio.

El Convenio formula criterios precisos a fin de remontar las barreras geográficas, económicas y organizativas para limitar el riesgo ante una EO. Los proveedores de las distintas instituciones aplican sus propios criterios desde una óptica discrecional. Por ejemplo, si un médico trabajaba en dos o más instituciones, favorecía este tipo de vínculos o, en casos de pacientes graves para las que no se obtenía una respuesta positiva para el traslado, los directivos o el secretario de salud intercedían para lograr su ingreso a una terapia intensiva, lo que da cuenta de que se privilegian los contactos personales a las normativas institucionales.

La AEO que necesita terapia intensiva está supeditada a la disponibilidad de espacios y de recursos físicos e institucionales, que son más limitados para la atención neonatal. Estrategias como el Convenio $y$ el Acuerdo ofrecen la posibilidad de organizar la infraestructura y los recursos de las distintas instituciones para brindar atención a la población que lo necesite. Sin embargo, no hay infraestructura suficiente para prestar servicios especializados a las mujeres y los neonatos. Las terapias habilitadas no disponen de los recursos humanos ni de los insumos suficientes, lo que impide su adecuada operación y conlleva la sobresaturación de aquellas unidades que sí cuentan con insumos, equipo y personal. Esto se ha puesto de manifiesto de manera más dramática durante la pandemia del Covid-19 que se está padeciendo en 2020.

Para aprovechar debidamente el Convenio, es necesario romper paradigmas en torno a la provisión de servicios para la AEO. Se documentó que los pro- 
veedores de salud de las instituciones de la seguridad social señalaron que no "expondrían a sus afiliadas" a otra institución cuya prestación de servicios consideraran de menor calidad. El marco normativo mexicano, integrado por las Guías de Práctica Clínica, las Normas Oficiales Mexicanas para la atención de la salud y los Programas de Acción Específicos, regula los procesos de la atención médica y tendría que evitar la variabilidad en la praxis por las decisiones diagnósticas y terapéuticas. La homologación de los procesos en cuanto a la calidad de la provisión de la atención en las distintas instituciones permitiría que el sector avance hacia una integración funcional, sobre la base de la mejora universal de la atención a la salud, con independencia de la afiliación.

\section{Conclusiones}

A partir de la instrumentación en 2009 del acceso universal a la AEO en México mediante el Convenio, y en 2011 con el Acuerdo, no se observa una clara evidencia de que los proveedores de servicios o las usuarias utilicen estos instrumentos para brindar/ recibir atención oportuna y expedita en el caso de una EO y, por lo consiguiente, tampoco la hay de que puedan superar las inequidades en el acceso entre las mujeres mexicanas, pese a la difusión que se otorgó en su momento al Decreto Presidencial a través de los medios de comunicación y a la existencia de la política pública de cero rechazo que reiteraba la esencia del Convenio; tampoco apoyándose, además, en la utilización de diversos oficios y misivas internas difundidas por las instituciones participantes dirigidas al personal de salud a lo largo de los años, con el fin de ampliar su utilización y difusión entre usuarias y proveedores. La provisión de AEO entre las distintas instituciones es limitada, y cuando ocurre, es en virtud de convenios y acuerdos locales o gestiones personales. Los aspectos limitantes de la provisión de AEO, como las barreras geográficas, económicas y organizativas, confirman la necesidad de operar en red para evitar demoras.

Otros aspectos limitantes ya mencionados, como las barreras socioculturales, dan cuenta de que en estados como Chiapas - en donde viven poblaciones hablantes de lenguas indígenas en una proporción municipal de hasta el $90 \%$ - los establecimientos que integrarían una red deben adecuarse para que en el continuo de la AEO haya pertinencia cultural; es decir, que se atiendan todas las necesidades de las mujeres y los neonatos. Una red para la AEO debe abarcar el primer nivel de atención como puerta de entrada ágil y disponer de una clara asociación con el resto de los niveles de atención del sistema de salud, sin importar qué institución preste los servicios. Es clave, al respecto, contar con un sistema de información que reúna los datos del padrón único de beneficiarias, y que lo utilicen todos los miembros de la red.

La AEO que necesita terapia intensiva está supeditada a la disponibilidad de espacios y de recursos físicos e institucionales, que son más limitados para la atención neonatal. Estrategias como el Convenio y el Acuerdo ofrecen la posibilidad de organizar la infraestructura y los recursos de las distintas instituciones para brindar atención a la población que lo necesite; sin embargo, no hay infraestructura suficiente para prestar servicios especializados a las mujeres y los neonatos. Las terapias habilitadas no disponen de los recursos humanos ni de los insumos suficientes, lo que impide su adecuada operación y conlleva la sobresaturación de aquellas unidades que sí cuentan con insumos, equipo y personal.

Cada una de las instituciones integrantes del sector tiene sus propios modelos de atención y brinda los servicios de AEO con una óptica particular, pese a las políticas y los acuerdos unificadores que cada una de ellas ha firmado. Estas políticas públicas no describen la conformación de las redes de servicios ni la provisión de atención en un marco de colaboración. De ello deriva la necesidad de configurar redes con características que respondan a las necesidades de las mujeres y los neonatos, asegurando el acceso universal a $-\mathrm{y}$ el continuo de - la AEO. Para coordinar el continuo de la AEO allí donde el acceso a cuidados especializados está implícito, es necesario planificar, inicialmente, la homologación de los distintos procesos y mecanismos de su operación, conformar los sistemas de referencia 
MARÍA Guadalupe Ramírez-Rojas, MARÍA GRACIELA FREYERMUTHENCISO, MARÍA BEATRIZ DUARTE-GÓMEZ

y ampliar la cobertura en la provisión de los servicios entre los hospitales que integren la red.

\section{Recomendaciones}

Los retos inherentes a la configuración y la aplicación de un enfoque de red son los siguientes:

1. Redefinir los mecanismos de coordinación que permitan asegurar el continuo de la $\mathrm{AEO}$, sobre la base de sistemas de referencia y contrarreferencia fortalecidos mediante la homologación de los procesos y los criterios de derivación de las AEO.

2. Incentivar la colaboración entre las distintas instituciones robusteciendo los recursos físicos y humanos de cada establecimiento, evitando la saturación de los centros hospitalarios de mayor capacidad resolutiva y favoreciendo una derivación estratégica y coordinada de los casos ajustados a las necesidades de las mujeres y los neonatos.

3. Establecer mecanismos que permitan evaluar la gestión de la calidad de la operatividad de las redes de servicios, en términos de oportunidad de las referencias y los resultados de la provisión de servicios, ya que dichos parámetros no son evaluados por las instituciones que participan del Convenio y del Acuerdo.

4. Medir y evaluar el desempeño de la provisión de servicios para la AEO desde la óptica del acceso universal y aplicar estrategias que fortalezcan a los integrantes de la red para ampliar, así, la cobertura y la capacidad resolutiva conjuntas. El éxito de la AEO oportuna de la red abatiría las visiones institucionales.

5. Consensuar entre las distintas instituciones respecto al Convenio, el Acuerdo o la resolución que adopten para brindar AEO. La coexistencia de múltiples acuerdos desvirtúa otras estrategias, incluso de mayor alcance, lo que ocasiona que los Convenios directos entre los hospitales limiten la operación de las políticas públicas que implican un mayor beneficio para la población, como es el caso del acceso universal a la AEO.

\section{Notas}

${ }^{1}$ Ha cambiado su nombre en diferentes administraciones: Programa IMSS-Coplamar (1979-1989), IMSS-Solidaridad (1989-2002), IMSS-Oportunidades (2002-2014), IMSS-Prospera (2014-2018) e IMSS-Bienestar (este último nombre es el que tiene desde diciembre de 2018).

${ }^{2}$ Resoluciones unilaterales dictadas por el Poder Ejecutivo con fines dirigidos a la administración pública y cuya resolución determina acciones de carácter público.

${ }^{3}$ Participan 466 establecimientos del segundo nivel de atención, acreditados para la AEO, de las instituciones incluidas en el Convenio. Según infraestructura, insumos y recursos humanos, estos pueden ser de resolución básica, media o alta. Son de resolución básica las unidades médicas hospitalarias para la atención y estabilización de pacientes obstétricas, y su ulterior referencia a un establecimiento con más recursos. De resolución media son los establecimientos que disponen de equipo médico especializado en ginecoobstetricia, anestesiología, pediatría, medicina interna, cirugía general y enfermería general; y además, de una unidad tocoquirúrgica, un cunero, hospitalización para obstetricia, un laboratorio clínico, un puesto de sangrado o servicios de transfusión e imagenología y, de manera opcional, terapia intermedia. Los establecimientos de alta resolución, además de lo que caracteriza a los establecimientos de resolución media, cuentan con la especialidad de medicina, terapia intensiva de adultos o neonatal.

${ }^{4}$ De acuerdo con estos organismos, las funciones de los establecimientos de AEO básica son: disponibilidad por la vía parenteral de medicamentos para la AEO (antibióticos, oxitócicos, anticonvulsivantes y antihipertensivos); personal calificado con conocimientos para realizar maniobras de extracción manual de la placenta, extracción del producto de la concepción retenido y asistencia de partos vaginales. Se especifica que estos establecimientos pueden ser centros de salud o centros hospitalarios. Los establecimientos de AEO integral son centros hospitalarios que tienen, además de las funciones descritas para los establecimientos de AEO básica, disponibilidad de quirófano con capacidad para realizar cirugías y capacidad para 
hacer transfusiones de sangre, y el personal de salud calificado para desarrollar dichas funciones. Tanto los establecimientos de AEO básica como los de AEO integral deben prestar sus servicios a las mujeres en caso de emergencia las 24 horas del día los 365 días del año, y en caso de que se requiriese, derivar a la paciente a un establecimiento con mayor capacidad resolutiva y disponer de los medios necesarios para evitar demoras.

${ }_{5}^{5}$ En el contexto chiapaneco existe la posibilidad de retenes o bloqueos en carreteras por cuestiones de diversa índole, lo cual podría ocasionar retrasos en los trayectos.

${ }^{6}$ De acuerdo con la Norma Oficial Mexicana NOM-004SSA3-2012, Del expediente clínico, la referencia y la contrarreferencia se definen como procesos médicos administrativos, mientras que en la definición de redes de servicios se trata de un sistema de referencia y contrarreferencia que permite el continuo en la red.

${ }^{7}$ Programa de afiliación al Seguro-Popular que incluye y enfoca la atención en la mujer embarazada.

${ }^{8}$ El IMSS-P presta servicios de salud del primero y segundo niveles de atención a la población que habita en zonas rurales y urbano-marginadas y carece de seguridad social. El universo de trabajo corresponde a los municipios y localidades que cuentan con establecimientos médicos.

\section{Referencias}

CIESAS (Centro de Investigaciones y Estudios Superiores en Antropología Social) e INMUJERES (Instituto Nacional de las Mujeres) (2010). "Monitoreo de la atención a las mujeres en servicios del Sector Salud. Atención Materna y Perinatal. Resultados por subíndices según institución”. México: CIESAS, INMUJERES. Disponible en http://web.inmujeres.gob.mx/ transparencia/archivos/estudios_opiniones/cuadernos/c29_o.pdf (consultado el 26 de junio de 2020).

Dávila, Carlos (1970). "Unidades de Cuidado Intensivo en América Latina”. En Boletín de la Oficina Sanitaria Panamericana, LXIX (1), 50-63.

DGCES (Dirección General de Educación y Calidad en Salud) (2013). Cédula de acreditación de establecimiento para la atención médica adherido al Convenio General de Cola- boración Interinstitucional para la Atención de la Emergencia Obstétrica. México: SSA. Disponible en http://calidad. salud.gob.mx/site/calidad/cedulas_acreditacion.html (consultado el 26 de junio de 2020).

DGED (Dirección General de Evaluación del Desempeño) (2009). Unidades de primer nivel de atención en los Servicios Estatales de Salud. Evaluación 2008. México: SSA.

Diario Oficial de la Federación (2012). "NOM-004SSA3-2012, Del expediente clínico”. 29 de junio de 2012, México.

Diario Oficial de la Federación (2013). "NOM-025SSA3-2013, Para la organización y funcionamiento de las unidades de cuidados intensivos". 17 de septiembre de 2013, México.

Diario Oficial de la Federación (2018). "Ley de Asociaciones Públicos Privadas", 16 de enero de 2012, México.

Flyvbjerg, Bent (2003). Making Social Science Matter. Cambridge: Cambridge University Press.

Gillies, Robin R., Stephen Shortell, D. Anderson, Julie Mitchell y Kerry Morgan (1993). "Conceptualizing and measuring Integration: Findings from the health Systems integration study". En Hospital and Health Services Administration, 38, 467-489.

GiménezMontiel,Gilberto(2012)."Elproblemadelageneralización en los estudios de caso". En Culturay RepresentacionesSociales, 7(13),40-62. Disponibleenhttp://www.scielo. org.mx/scielo.php?script=sci_arttext\&pid=S2007$81102012000200002 \&$ \&lng=es\&nrm=iso

Herrera Beltrán, José Luis, Juan Felipe Conde Roa y Olga Lucía Pinzón Espitia (2017). "Evaluación de una Red Integrada de Servicios de Salud en Colombia”. En Archivos de Medicina, 13(3):14.

Hurtado Ochoterena, Claudia A. y Norma A. Matías Juan (2005). "Historia de la vacunación en México". En Revista Mexicana de Puericultura y Pediatría, 13(74), 48-52.

IMSS (Instituto Mexicano del Seguro Social) (2006). “Acuerdo 126/2006. Dirección de Prestaciones Médicas. 'Se autoriza que se otorgue atención médica en hospitales de 2 do. y 3er. nivel a las mujeres embarazadas que se identifiquen como beneficiarias del Programa IMSS Oportunidades"'. México: IMSS. Disponible en http:// www.imss.gob.mx/conoce-al-imss/acuerdos2006 (consultado el 26 de junio de 2020). 
MARÍA GuAdalupe Ramírez-Rojas, MARÍA GRACIELA FREYERMUTHENCISO, MARÍA BEATRIZ DUARTE-GÓMEZ

IMSS (Instituto Mexicano del Seguro Social) (2016) "Acuerdo mediante el cual se establecen las Reglas de Operación del Programa IMSS-Prospera para el ejercicio fiscal 2017". En Diario Oficial de la Federación, 22 de diciembre. Disponible en http://www.dof.gob.mx/ nota_detalle.php?codigo=5466678\&\&fecha=22/12/2016 Exprint=true (consultado el 26 de junio de 2020).

Londoño, Juan y Julio Frenk (1997). "Pluralismo estructurado: hacia un modelo innovador para la reforma de los sistemas de salud en América Latina". En Oficina del Economista Jefe. Documento de trabajo 353. BID. Disponible en https://www.researchgate. net/publication/23531504_Pluralismo_estructurado_Hacia_un_modelo_innovador_para_la_reforma_de_los_sistemas_de_salud_en_America_Latina (consultado el 26 de junio de 2020).

Mesa-Lago, Carmelo (2005). Las reformas de salud en América Latina y el Caribe: su impacto en los principios de seguridad social. Santiago de Chile: CEPAL.

MINSALUD (Ministerio de Salud y Protección Social) (2016). Redes integrales de prestadores de servicios de salud. Lineamientos para el proceso de conformación, organización, gestión, seguimiento y evaluación. Bogotá: MINSALUD.

OCDE (Organización para la Cooperación y el Desarrollo Económicos) (2016). Estudios de la OCDE sobre los Sistemas de Salud: México 2016. París: OCDE.

OMM (Observatorio de Mortalidad Materna en México) (2011). Primer monitoreo. Atención de emergencias obstétricas en Tabasco a través del Convenio General de Colaboración Interinstitucional. Ciudad de México: CIESAS. Disponible en https:/omm.org.mx/wp-content/uploads/2020/04/ Tabasco_CAEO-2011_S\%C3\%ADntesis.pdf (consultado el 26 de junio de 2020).

OMM(Observatorio de Mortalidad Maternaen México) (2013). Monitoring the General Agreement of Interangency Collaboration for Emergency Obstetric Care in Chiapas. Second Monitoring. México: CIESAS. Disponible en https:/omm.org.mx/wp-content/uploads/2020/04/ Chiapas_CAEO-2012_S\%C3\%ADntesis-englishversion.pdf (consultado el 27 de agosto de 2020).

OMM (Observatorio de Mortalidad Materna en México) (2016). Tercer monitoreo 2015. Convenio General de Colaboración Interinstitucional para la Atención de la
Emergencia Obstétrica. Región Chiapas: Tuxtla Gutiérrez y Tapachula. México: CIESAS. Disponible en https:// omm.org.mx/wp-content/uploads/2020/04/Chiapas_CAEO-2015_S\%C3\%ADntesis.pdf (consultado el 26 de junio de 2020).

OMM (Observatorio de Mortalidad Materna en México) (2018). Numeralia 2016. Mortalidad materna en México. México: CIESAS. Disponible en https:/omm.org. mx/wp-content/uploads/2020/04/Numeralia-2016. pdf (consultado el 26 de junio de 2020).

OMS (Organización Mundial de la Salud), UNFPA (Fondo de Población de las Naciones Unidas), UNICEF (Fondo de las Naciones Unidas para la Infancia) y AMDD (Averting Maternal Death and Disability) (2011). Seguimiento de los servicios obstétricos de urgencia. Ginebra: OMS.

OPS (Organización Panamericana de la Salud) (2010). Metodología de Gestión Productiva de los Servicios de Salud. Introducción y Generalidades. Washington, D.C.: OPS.

Ramírez Rojas, María Guadalupe y María Graciela Freyermuth Enciso (2013). "Atención de emergencias obstétricas a través del Convenio General de Colaboración Interinstitucional. Entrevistas a usuarias beneficiadas". En Revista CONAMED, 18(3), 100-103.

SSA (Secretaría de Salud) (2009). "Convenio General de Colaboración que celebran la Secretaría de Salud, el Instituto Mexicano del Seguro Social y el Instituto de Seguridad y Servicios Sociales de los Trabajadores del Estado, para la Atención de las Emergencias Obstétricas, el 28 de mayo de 2009". México: SSA. Disponible en http:// cnegsr.salud.gob.mx/contenidos/descargas/SMP/ConvenioEO.pdf (consultado el 26 de junio de 2020).

SSA (Secretaría de Salud) (2017). "Acuerdo General de Colaboración para el Intercambio de Servicios, celebrado por la Secretaría de Salud, el Instituto Mexicano del Seguro Social y el Instituto de Seguridad y Servicios Sociales de los Trabajadores del Estado, Institutos Nacionales, Hospitales Federales de Referencia y Hospitales Regionales de Alta Especialidad, firmado el 12 de mayo del 201l”. México: SSA.

Tello Delgado, María Paulina (2007). Cuidado integral al recién nacido pretérmino menor de 1500 gr de peso, un reto para enfermería. Chiclayo, Perú: Universidad Católica San- 
to Toribio de Mogrovejo. Disponible en http://www. reeme.arizona.edu/materials/cuidado\%20inegral $\% 20$ rnpt\%202.pdf (consultado el 26 de junio de 2020).

UNFPA (Fondo de Población de las Naciones Unidas) (2002). "Programa AMDD (Evitar la Muerte y Discapacidad Materna), Sistema de aprendizaje a distancia sobre cuestiones de población. Curso 6 . Reducción del número de defunciones maternas: Selección de prioridades, registro del progreso. Módulo 2. Utilización de indicadores para determinar el progreso en la reducción del número de defunciones maternas". Italia: Universidad de Columbia.

Figura 1. Propuesta de red para la AEO

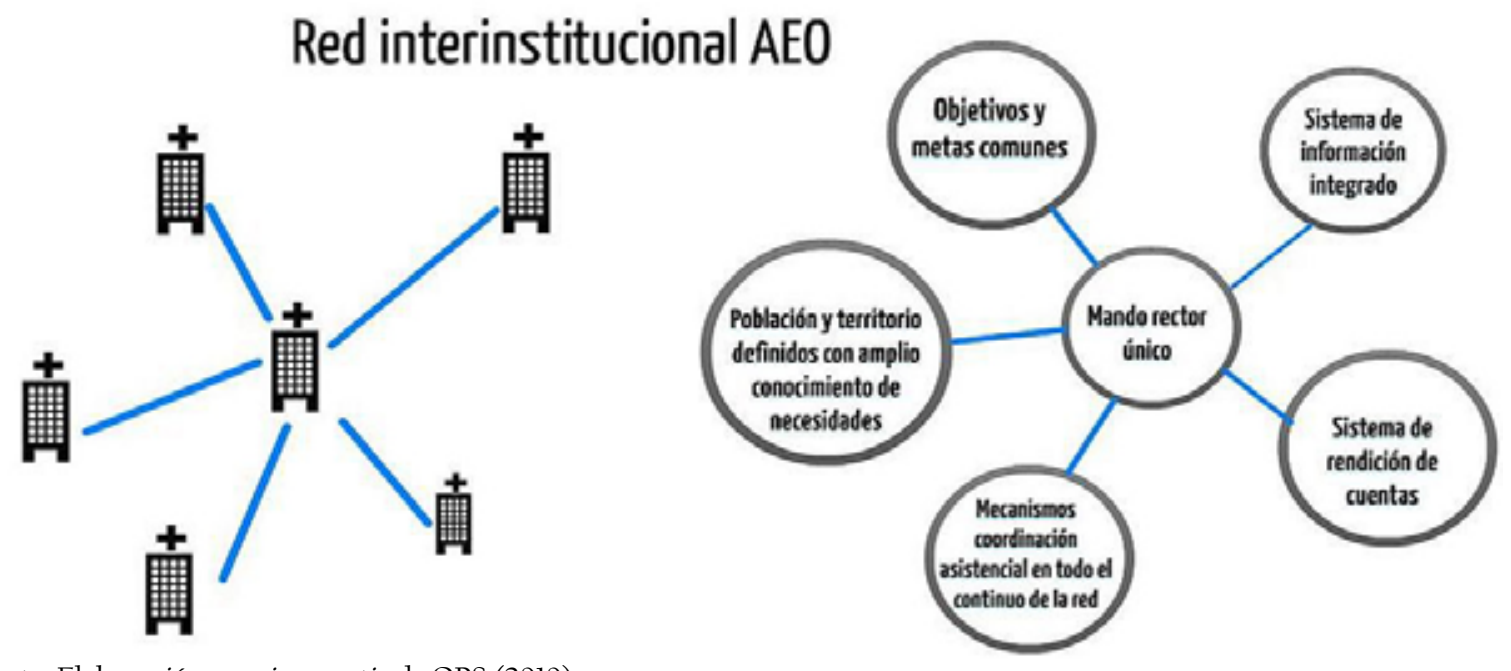

Fuente: Elaboración propia a partir de OPS (2010).

Figura 2. Atención de las emergencias obstétricas en México

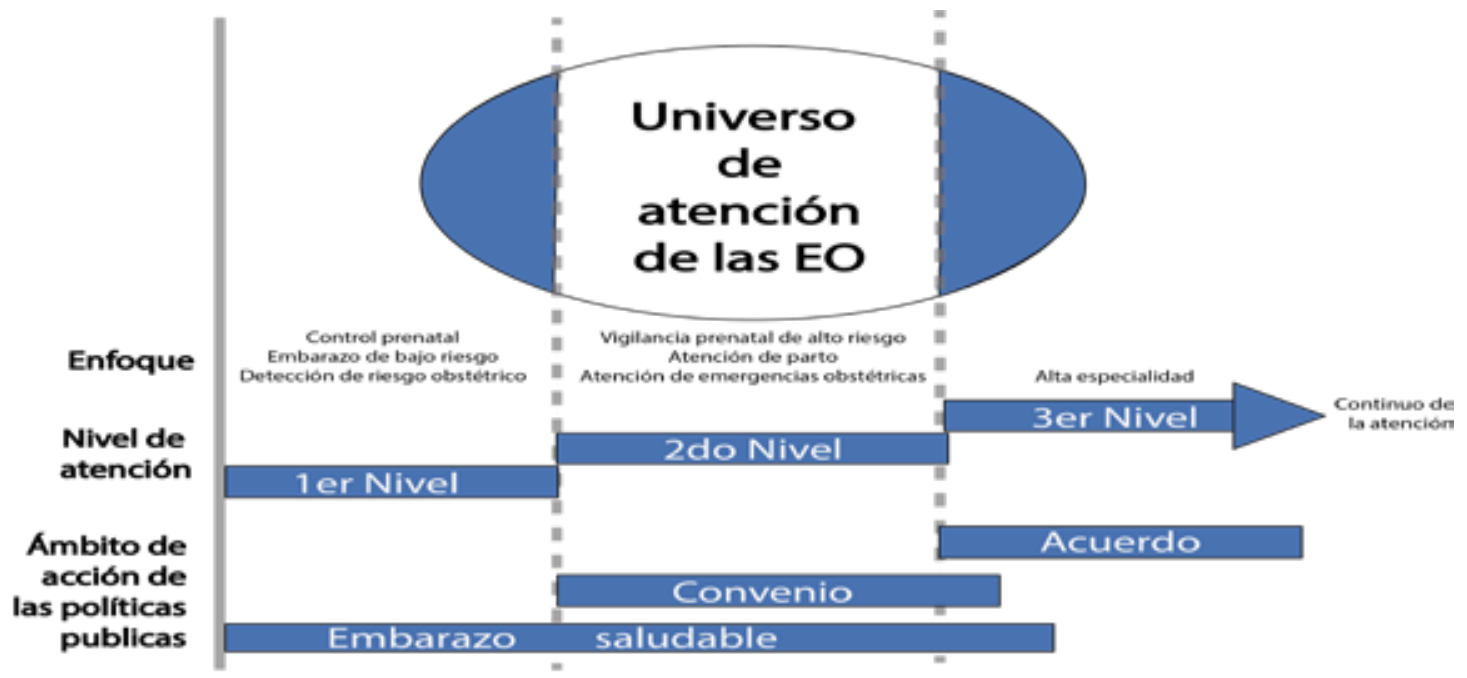

Fuente: Elaboración propia, 2015. 
Figura 3. Referencias para la AEO en Chiapas, 2015

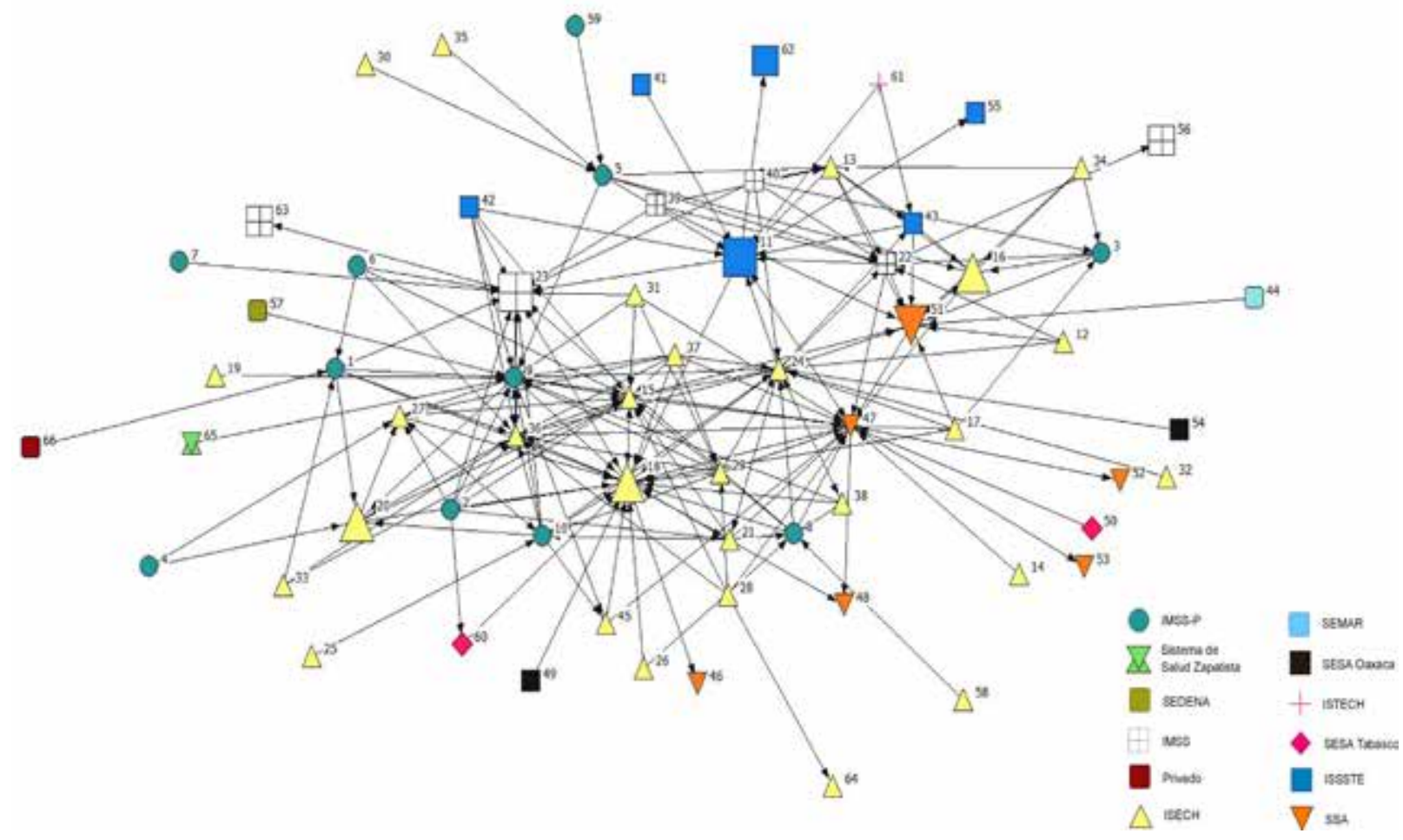

Fuente: Elaboración propia, 2015. Nodos (con * aquellos ubicados en la Ciudad de México, ${ }^{* *}$ en Oaxaca y *** en Tabasco): 1. Hospital Rural (HR) Altamirano, IMSS-P, 2. HR Bochil, IMSS-P, 3. HR Mapastepec, IMSS-P, 4. HR Guadalupe Tepeyac, IMSS-P, 5. HR Motozintla, IMSS-P, 6. HR Ocosingo, IMSS-P, 7. HR Benemérito de las Américas, IMSS-P, 8. HR Ocozocoautla, IMSS-P, 9. HR San Felipe Ecatepec, IMSS-P, 10. HR Venustiano Carranza, IMSS-P, 1l. HG Belisario Domínguez, Tuxtla Gutiérrez, ISSSTE, 12. HG Juárez, Arriaga, ISECH, 13. HG Huixtla, ISECH, 14. HG Palenque, ISECH, 15. H. de la Mujer, San Cristóbal de Las Casas, ISECH, 16. HG Tapachula, ISECH, 17. HG Dr. Juan C. Corzo, Tonalá, ISECH, 18. HG Dr. Rafael Pascasio Gamboa, Tuxtla Gutiérrez, ISECH, 19. HG Yajalón, ISECH, 20. H. de la Mujer, Comitán, ISECH, 21. HG Villaflores, ISECH, 22. Hospital General de Zona IMSS (HGZMF) I Tapachula, IMSS, 23. HGZ 2 Tuxtla Gutiérrez, IMSS, 24. Hospital Básico Comunitario (HBC) Acala, ISECH, 25. Hospital Básico Comunitario (HBC) Ángel Albino Corzo, ISECH, 26. HBC Cintalapa de Figueroa, ISECH, 27. HG María Ignacia Gandulfo, Comitán, ISECH, 28. HBC Chalchihuitán, ISECH, 29. HBC San Juan Chamula, ISECH, 30. HBC Frontera Comalapa, ISECH, 31. HBC Larráinzar, ISECH, 32. HBC Las Margaritas, ISECH, 33. HBC Ocosingo, ISECH, 34. HBC Pijijiapan, ISECH, 35. HBC Porvenir, ISECH, 36. H. Culturas, San Cristóbal de Las Casas, ISECH, 37. HBC Teopisca, ISECH, 38. HBC Revolución Mexicana, ISECH, 39. HGSMF 19 Huixtla, IMSS, 40. HGSMF 15 Tonalá, IMSS, 41. Clínica Hospital (CH) Comitán de Domínguez, ISSSTE, 42. CH San Cristóbal de Las Casas, ISSSTE, 43. CH DR. Roberto Nettel F., ISSSTE, 44. Sanatorio Naval de Puerto Chiapas, SEMAR, 45. HE Gómez Maza, ISECH, 46. Instituto Nacional de Perinatología*, SSA, 47. HEP Tuxtla Gutiérrez, SSA, 48. H. Infantil Federico Gómez*, SSA, 49. HG Arriaga**, Oaxaca, SESA, 50. H. del Niño***, Tabasco, SESA, 5l. HRAE Ciudad Salud, ISECH, 52. Instituto Nacional de Cardiología*, SSA, 53. Instituto Nacional de Pediatría*, SSA, 54. HG Salina Cruz**, Oaxaca, SESA, 55. HG Adolfo López Mateos*, ISSSTE, 56. CMN S. XXI H. Pediatría*, IMSS, 57. H. Militar Regional de Tuxtla Gutiérrez, SEDENA, 58. HBC Berriozábal, ISECH, 59. Centro de Atención Rural Obstétrica (CARO) Porvenir, IMSS-P, 60. HRAE Rovirosa***, Tabasco, SESA, 6l. HE Vida Mejor, Instituto de Seguridad Social de los Trabajadores del Estado de Chiapas (ISTECH), 62. H. Regional General Ignacio Zaragoza *, ISSSTE, 63. H. Ginecoobstetricia 4 *, IMSS, 64. H. de la Mujer, San Cristóbal de Las Casas, ISECH, 65. Clínica Guadalupana Oventic, Sistema de Salud Zapatista, 66. H. las Mañanitas Ocosingo, Privado. 
Tabla 1. Disponibilidad de terapias intensivas en establecimientos hospitalarios según su capacidad resolutiva y su inclusión en el Convenio

\begin{tabular}{|c|c|c|c|c|c|c|}
\hline \multicolumn{4}{|c|}{ Convenio } & \multicolumn{3}{|c|}{ No participa en el Convenio } \\
\hline UCIA y UCIN & UCIA & UCIN & $\begin{array}{c}\text { Terapia } \\
\text { intermedia }\end{array}$ & UCIA & UCIN & $\begin{array}{c}\text { Terapia } \\
\text { intermedia }\end{array}$ \\
\hline $\begin{array}{l}\text { ISECH*: } \\
\text { HG } \\
\text { Tapachula, } \\
\text { IMSS**: } \\
\text { HGZ 2 Tuxtla } \\
\text { Gutiérrez, } \\
\text { HGZMF 1 } \\
\text { Tapachula }\end{array}$ & $\begin{array}{l}\text { ISSSTE*: } \\
\text { HG Dr. } \\
\text { Belisario } \\
\text { Domínguez }\end{array}$ & $\begin{array}{l}\text { ISECH: } \\
\text { H. de la Mujer, } \\
\text { San Cristóbal } \\
\text { de Las Casas**, } \\
\text { H. Regional Dr. } \\
\text { Rafael Pascasio } \\
\text { Gamboa*, H. } \\
\text { de la Mujer } \\
\text { Comitán* }\end{array}$ & $\begin{array}{l}\text { IMSS**: } \\
\text { HGZMF } 1 \\
\text { Tapachula; } \\
\text { ISECH*: } \\
\text { HR Dr. Rafael } \\
\text { Pascasio } \\
\text { Gamboa }\end{array}$ & $\begin{array}{l}\text { ISECH: HG María } \\
\text { Ignacia Comitán, HE } \\
\text { Gómez Maza, H. de } \\
\text { las Culturas; } \\
\text { SSA: HRAE Ciudad } \\
\text { Salud, Tapachula; } \\
\text { ISSSTE: CH Dr. } \\
\text { Roberto Nettel } \\
\text { Flores }\end{array}$ & $\begin{array}{l}\text { SSA: HEP } \\
\text { Tuxtla } \\
\text { Gutiérrez }\end{array}$ & $\begin{array}{l}\text { SSA: HEP } \\
\text { Tuxtla } \\
\text { Gutiérrez }\end{array}$ \\
\hline $\begin{array}{l}\text { ISECH*: } \\
\text { HG } \\
\text { Tapachula, } \\
\text { IMSS**: } \\
\text { HGZ 2 Tuxtla } \\
\text { Gutiérrez, } \\
\text { HGZMF } 1 \\
\text { Tapachula }\end{array}$ & $\begin{array}{l}\text { ISSSTE*: } \\
\text { HG Dr. } \\
\text { Belisario } \\
\text { Domínguez }\end{array}$ & $\begin{array}{l}\text { ISECH: } \\
\text { H. de la Mujer, } \\
\text { San Cristóbal } \\
\text { de Las Casas**, } \\
\text { H. Regional Dr. } \\
\text { Rafael Pascasio } \\
\text { Gamboa*, H. } \\
\text { de la Mujer } \\
\text { Comitán* }\end{array}$ & $\begin{array}{l}\text { IMSS**: } \\
\text { HGZMF } 1 \\
\text { Tapachula; } \\
\text { ISECH*: } \\
\text { HR Dr. Rafael } \\
\text { Pascasio } \\
\text { Gamboa }\end{array}$ & $\begin{array}{l}\text { ISECH: HG María } \\
\text { Ignacia Comitán, HE } \\
\text { Gómez Maza, H. de } \\
\text { las Culturas; } \\
\text { SSA: HRAE Ciudad } \\
\text { Salud, Tapachula; } \\
\text { ISSSTE: CH Dr. } \\
\text { Roberto Nettel } \\
\text { Flores }\end{array}$ & $\begin{array}{l}\text { SSA: HEP } \\
\text { Tuxtla } \\
\text { Gutiérrez }\end{array}$ & $\begin{array}{l}\text { SSA: HEP } \\
\text { Tuxtla } \\
\text { Gutiérrez }\end{array}$ \\
\hline
\end{tabular}

Fuente: Elaboración propia, 2015 a partir de información del Convenio, y el Monitoreos al Convenio (OMM).

* Capacidad resolutiva alta.

** Capacidad resolutiva media según el Convenio. 


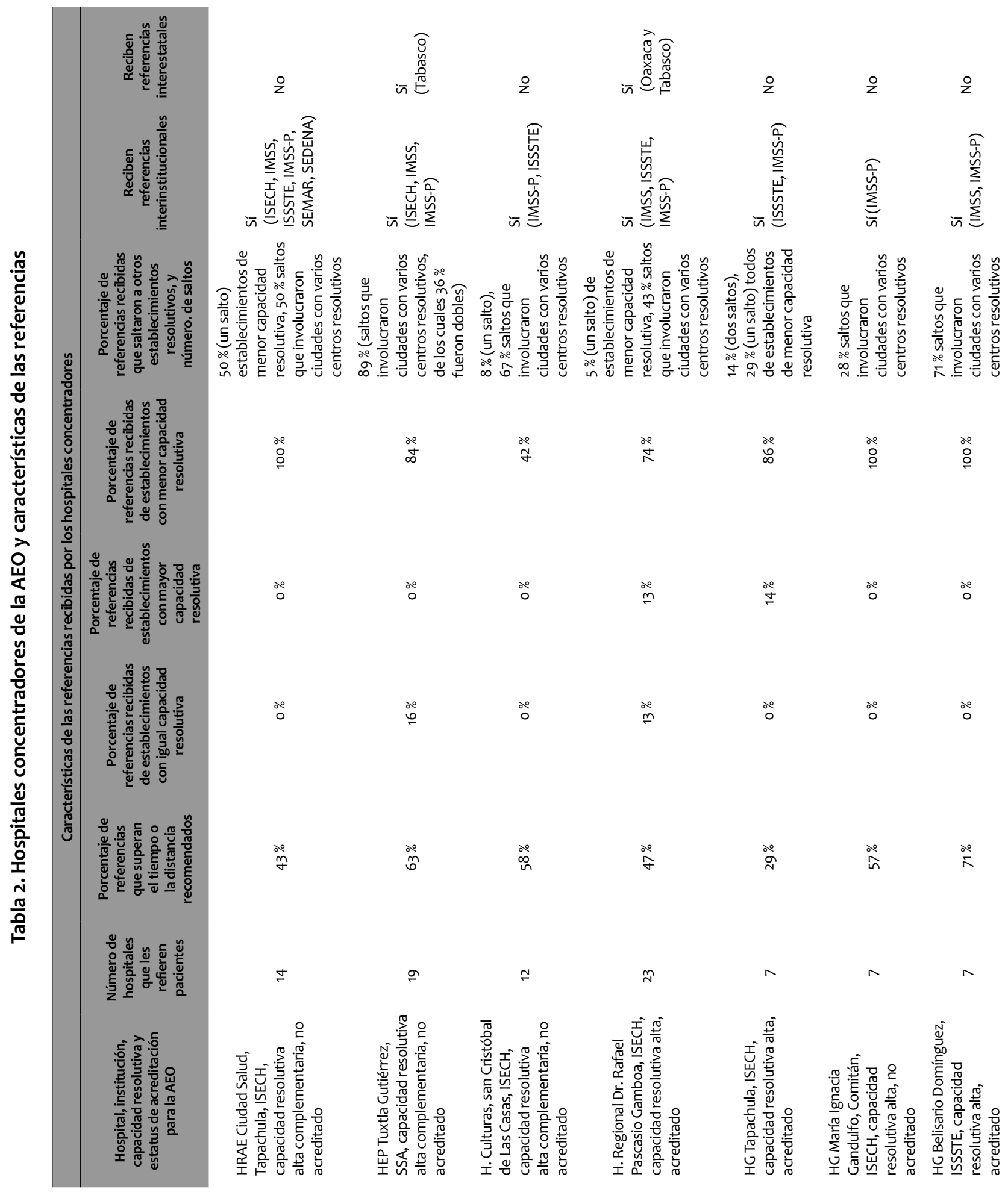




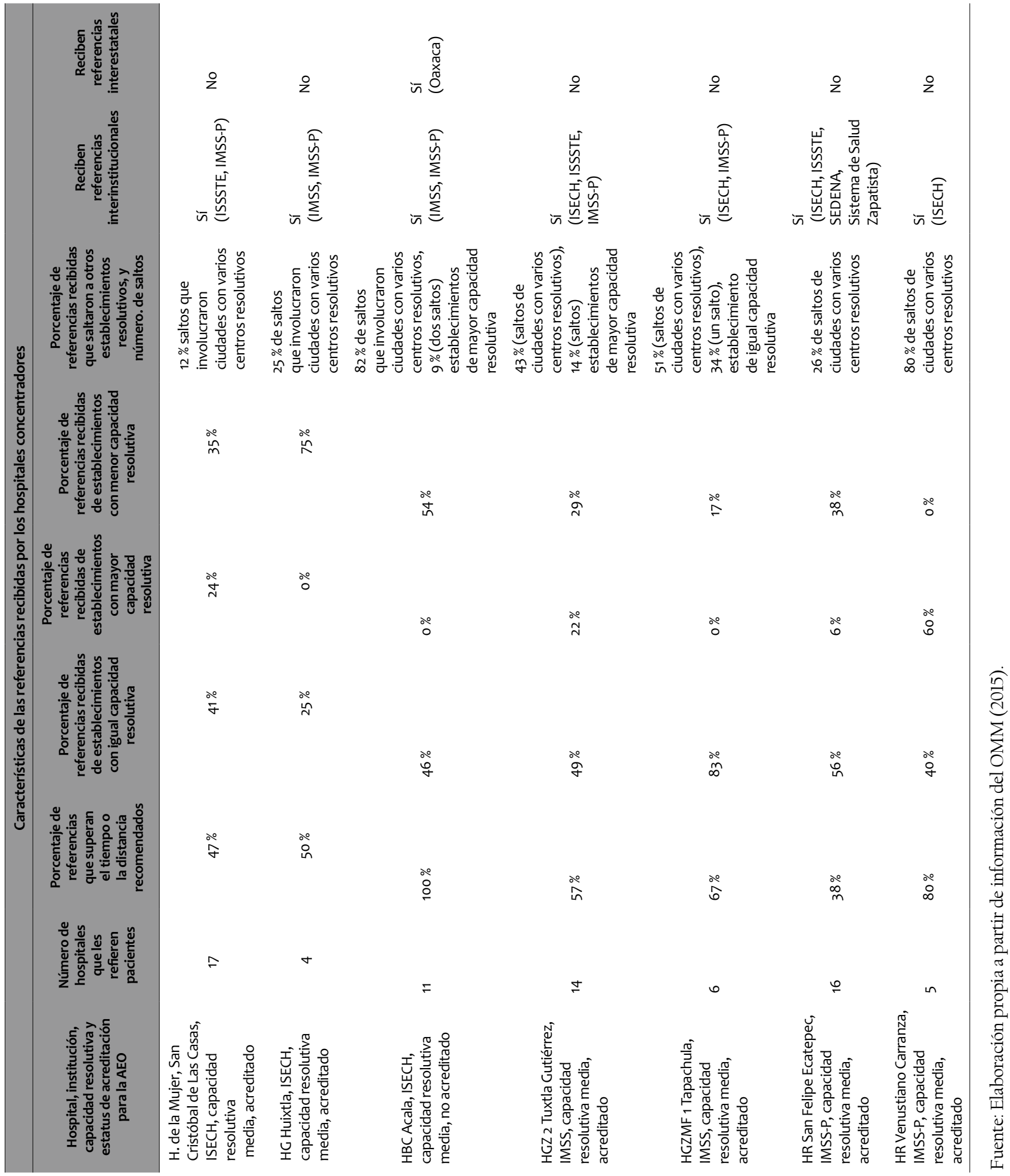


Tabla 3. Distancia y tiempo de recorrido de los hospitales que refieren usuarias al HR Venustiano Carranza, IMSS-P

\begin{tabular}{|c|c|c|c|}
\hline $\begin{array}{l}\text { Establecimiento que hace } \\
\text { la referencia }\end{array}$ & Distancia & Tiempo & Posibilidades de referencia más cercanas \\
\hline $\begin{array}{l}\text { H. Regional Dr. Rafael } \\
\text { Pascasio Gamboa, ISECH }\end{array}$ & $88.2 \mathrm{~km}$ & $1 \mathrm{~h} 39 \mathrm{~min}$ & \\
\hline HG Villaflores, ISECH & $146 \mathrm{~km}$ & $2 \mathrm{~h} 35 \mathrm{~min}$ & $\begin{array}{l}\text { Tuxtla Gutiérrez: HGZ } 2 \text { Tuxtla Gutiérrez, } \\
\text { IMSS, HG Belisario Domínguez, } \\
\text { ISSSTE y HEP Tuxtla Gutiérrez, SSA }\end{array}$ \\
\hline HBC Acala, ISECH & $52 \mathrm{~km}$ & $1 \mathrm{~h} 7 \mathrm{~min}$ & \\
\hline $\begin{array}{l}\text { HBC Ángel Albino Corzo, } \\
\text { ISECH }\end{array}$ & $194 \mathrm{~km}$ & $3 \mathrm{~h} 17 \mathrm{~min}$ & $\begin{array}{l}\text { Villaflores: HR Villaflores, Tuxtla Gutiérrez: } \\
\text { HGZ } 2 \text { Tuxtla Gutiérrez, IMSS, } \\
\text { HG Belisario Domínguez ISSSTE y HEP Tuxtla } \\
\text { Gutiérrez, SSA }\end{array}$ \\
\hline $\begin{array}{l}\text { HG María Ignacia Gandulfo, } \\
\text { Comitán, ISECH }\end{array}$ & $79.4 \mathrm{~km}$ & $1 \mathrm{~h} 45 \mathrm{~min}$ & $\begin{array}{l}\text { San Cristóbal de Las Casas: H. de las } \\
\text { Culturas y H. de la Mujer, ISECH, HR San } \\
\text { Felipe Ecatepec, IMSS-P, CH San Cristóbal } \\
\text { Ecatepec, y en Comitán: H. de la Mujer, } \\
\text { Comitán, ISSSTE }\end{array}$ \\
\hline
\end{tabular}

Fuente: Elaboración propia., a partir de información del OMM (2015). 